Braun $\cdot$ Kooperation im Unternehmen 


\section{Wolfram Braun}

Kooperation
im Unternehmen

Organisation und

Steuerung von Innovationen 
Dr. Wolfram Braun ist Universitätsprofessor für Allgemeine Betriebswirtschaftslehre und Unternehmensführung am Fachbereich Wirtschaftswissenschaft der Universität - GH - Wuppertal.

CIP-Titelaufnahme der Deutschen Bibliothek

\section{Braun, Wolfram:}

Kooperation im Unternehmen : Organisation und Steuerung von

Innovationen / Wolfram Braun. - Wiesbaden : Gabler, 1991

Der Gabler Verlag ist ein Unternehmen der Verlagsgruppe Bertelsmann International.

(C) Betriebswirtschaftlicher Verlag Dr. Th. Gabler, Wiesbaden 1991

Lektorat: Gudrun Knöll

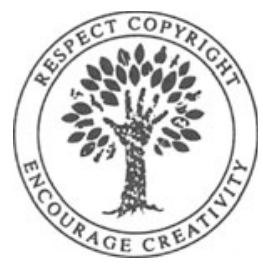

Das Werk einschließlich aller seiner Teile ist urheberrechtlich geschützt. Jede Verwertung außerhalb der engen Grenzen des Urheberrechtsgesetzes ist ohne Zustimmung des Verlags unzulässig und strafbar. Das gilt insbesondere für Vervielfältigungen, Übersetzungen, Mikroverfilmungen und die Einspeicherung und Verarbeitung in elektronischen Systemen.

ISBN-13:978-3-409-13650-1

e-ISBN-13:978-3-322-89334-5

DOI: 10.1007/978-3-322-89334-5 


\section{Vorwort}

Die Innovationsfähigkeit von Unternehmen und die Effizienz der Forschung und Entwicklung sind zu wesentlichen Wettbewerbsfaktoren geworden und determinieren Richtung und Verlauf des technologischen Fortschritts und der gesamtwirtschaftlichen Entwicklung. Über die Realisierung strategischer Positionen im Innovationswettbewerb der verschiedenen Branchen entscheiden nicht zuletzt eine wirtschaftlich effiziente Organisation und Steuerung der Durchführung von Innovationsprojekten und die Forschungs- und Entwicklungskosten. Die laterale Kooperation der maßgeblich an Innovationsprojekten beteiligten Funktionsbereiche: Forschung und Entwicklung, Marketing und Produktion, mit weitgehend eigenverantwortlicher Steuerung der Projektdurchführung und Abstimmung von Funktionsbereichsstrategien, gehört zu jenen Organisationsformen, die in der Unternehmenspraxis zunehmend an Bedeutung gewinnen und in den Mittelpunkt betriebswirtschaftlicher Organisations- und Innovationsforschung rücken. Eine ökonomisch effiziente Organisation lateraler Kooperation, die Koordination von Funktionsbereichsstrategien und die Steuerung der Kooperations- und Innovationsprozesse mit betriebswirtschaftlichen Steuerungsinstrumenten, die Evaluation des Projekterfolgs und der Leistungsbeiträge der kooperierenden Funktionsbereiche sowie die Einrichtung effektiver Anreiz- und Belohnungssysteme für kooperatives und innovatives Verhalten, organisatorische und personelle Maßnahmen zur Lösung von Abstimmungsproblemen und Kooperationskonflikten und eine koordinationskostenminimale Durchführung von Innovationsprojekten - das sind zentrale Problemschwerpunkte unternehmerischer Innovationsforschung. Kooperationsmanagement hat die Bewältigung der organisatorischen und personellen Probleme bei der Durchführung von Innovationsprojekten zur Aufgabe. Die mikroökonomische und empirische Analyse der Voraussetzungen und Konsequenzen der Organisation und Steuerung von Innovationen sowie die Konzeptualisierung eines mikroökonomisch fundierten Kooperationsmanagements ist Gegenstand dieser Monographie.

Teil A der nachfolgenden Ausführungen behandelt die Ergebnisse der betriebswirtschaftlichen Organisations- und Innovationsforschung, die sich mit der Organisation von Forschungs- und Entwicklungsaktivitäten und ihrer funktionalen Integration in den betrieblichen Leistungsprozeß befaßt. Auf dieser Grundlage wird in Teil B das Konzept eines mikroökonomisch fundierten Kooperationsma- 
nagements und seine empirische Anwendung auf Probleme einer ökonomisch effizienten Organisation und Steuerung der Durchführung von Innovationsprojekten dargestellt. Gegenstand des ersten Kapitels sind mikroökonomische Modelle der Organisation und Steuerung lateraler Kooperationsbeziehungen zwischen Forschung und Entwicklung, Marketing und Produktion. Die Ergebnisse empirischer Fallstudien zur Organisation und Durchführung von Innovationsprojekten in Unternehmen der Branchen: Elektronik, Pharma und Elektrotechnik sowie die Ergebnisse mikroökonomischer Analyse und der mit diesen Ergebnissen und den Modellen begründeten Anwendung des Konzepts eines mikroökonomisch fundierten Kooperationsmanagements auf die Lösung praktischer Abstimmungs- und Koordinationsprobleme werden in den nachfolgenden Kapiteln behandelt.

Die Anwendung der mikroökonomischen Theorie auf Organisations- und Koordinationsprobleme ist ein noch relativ junges Forschungsgebiet der Betriebswirtschaftslehre. Deshalb werden die Konzepte und Problemstellungen mikroökonomischer Organisationstheorie unter besonderer Berücksichtigung der Probleme lateraler Kooperationsorganisation und -steuerung, im Anhang unter dem Stichwort "Mikroökonomische Organisationstheorie" besprochen.

Die Monographie ist Bestandteil eines Forschungsprogramms, das die Anwendung der mikroökonomischen Theorie auf betriebswirtschaftliche Problemstellungen zum Gegenstand hat. Die Veröffentlichungen: "Die Organisation ökonomischer Aktivitäten" (Gabler Verlag: Wiesbaden 1987), "Die Ökonomik der Unternehmung" (2. Aufl., Gabler Verlag: Wiesbaden 1989) und "Unternehmenspolitik im europäischen Binnenmarkt" (Gabler Verlag: Wiesbaden 1990) behandeln zusätzliche organisationale, funktionale und wettbewerbsbezogene Dimensionen, die in dieser Monographie nur angesprochen aber nicht ausgeführt werden. Die Anwendung des Forschungsprogramms auf die empirische Analyse von Kooperationsproblemen bei der Organisation und Durchführung von Innovationen in Unternehmen verschiedener Branchen war Teil eines Forschungsprojektes, das ich mit Herrn Kollegen Wunderer durchführen konnte. Ihm und seinem Mitarbeiter, Herrn Walser, möchte ich an dieser Stelle besonders herzlich danken. 


\section{Inhaltsverzeichnis}

Vorwort $\ldots \ldots \ldots \ldots \ldots \ldots \ldots \ldots \ldots \ldots \ldots \ldots \ldots \ldots \ldots \ldots \ldots \ldots \ldots \ldots \ldots$

\section{Teil A}

Organisation und Integration der Forschung und Entwicklung $\ldots \ldots \ldots \ldots \ldots$

I. Innovation und Forschung und Entwicklung $\ldots \ldots \ldots \ldots \ldots \ldots \ldots$

1. Innovation und Innovationsproze $\ldots \ldots \ldots \ldots \ldots \ldots \ldots \ldots \ldots \ldots$

1.1. Innovation . . . . . . . .

1.2. Innovationsarten ......................... 4

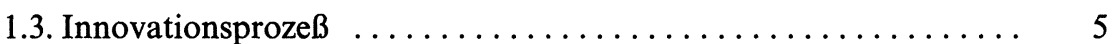

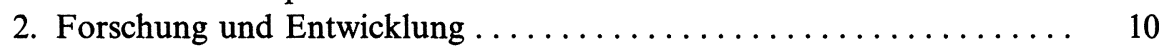

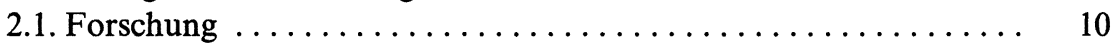

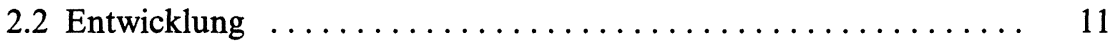

II. Umfeld betrieblicher Forschung und Entwicklung $\ldots \ldots \ldots \ldots \ldots \ldots \ldots$

1. Innovations- und Wettbewerbsprozesse ............... 11

2. Unternehmensinterne Bestimmungsfaktoren für Innovationen ..... 22

3. Rahmenbedingungen betrieblicher Forschung und Entwicklung ..... 24

III. Organisation der Forschung und Entwicklung $\ldots \ldots \ldots \ldots \ldots \ldots \ldots$

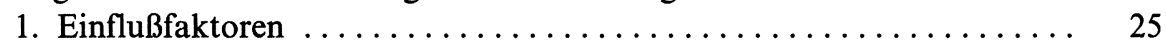

2. Interne und externe Organisation des FuE-Bereiches .......... 26

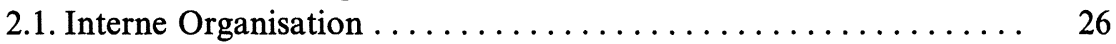

2.2. Integration des FuE-Bereiches in die Unternehmensorganisation . 32

2.3. Struktur und Strategien der Organisation von FuE-Aktivitäten ... 38

3. Spezielle Organisationsformen $\ldots \ldots \ldots \ldots \ldots \ldots \ldots \ldots \ldots \ldots$

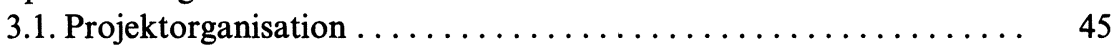

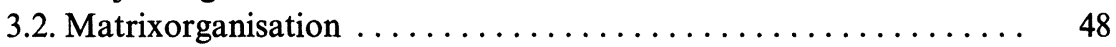

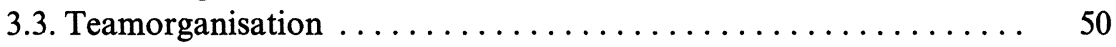

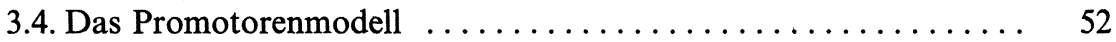

IV. Laterale Kooperation mit Forschungs- und Entwicklungsabteilungen ... 55

1. Produktion und Forschung und Entwicklung ............. 57

2. Marketing und Forschung und Entwicklung $\ldots \ldots \ldots \ldots \ldots \ldots \ldots$

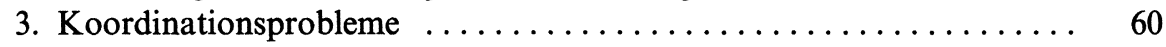

V. Effizienz und Evaluation betrieblicher Forschung und Entwicklung .... 63

1. Effizienzkriterien und Evaluationsverfahren ............... 64 
1.1. Inputmaße $\ldots \ldots \ldots \ldots \ldots \ldots \ldots \ldots \ldots \ldots \ldots \ldots \ldots \ldots \ldots \ldots \ldots, \quad 65$

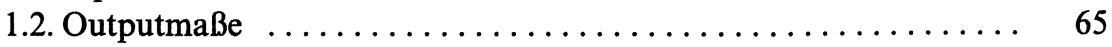

1.3. Verknüpfung von Input- und Outputmaßen $\ldots \ldots \ldots \ldots \ldots \ldots 68$

1.4. Produktionsfunktion der Forschung und Entwicklung ........ 69

1.5. Mehrdimensionale Evaluationsverfahren $\ldots \ldots \ldots \ldots \ldots \ldots \ldots, 70$

2. FuE-Controlling $\ldots \ldots \ldots \ldots \ldots \ldots \ldots \ldots \ldots \ldots \ldots \ldots \ldots \ldots \ldots \ldots \ldots, 73$

\section{Teil B}

Laterale Kooperation zwischen Forschung und Entwicklung, Marketing und Produktion - Mikroökonomische Theorie und ihre empirische Anwendung ...

\section{Kapitel 1}

Organisation und Steuerung lateraler Kooperationsbeziehungen Angewandte Mikoökonomie $\ldots \ldots \ldots \ldots \ldots \ldots \ldots \ldots \ldots \ldots . . . \ldots \ldots$

I. Grundfragen mikroökonomischer Analyse und Gestaltung lateraler Kooperation ................................. 82

1. Schließen sich laterale Kooperation und betriebswirtschaftliche Steuerung aus? ............................... 84

2. Kooperationssteuerung $\ldots \ldots \ldots \ldots \ldots \ldots \ldots \ldots \ldots \ldots \ldots \ldots, 85$

II. Kooperationsorganisation und Steuerungsmodelle $\ldots \ldots \ldots \ldots \ldots \ldots$

III. Zentrale versus dezentrale Steuerung lateraler Kooperationsbeziehungen . 101

1. Laterale Kooperation ohne zentrale Steuerung .............. 101

2. Laterale Kooperation mit zentraler Steuerung .............. 104

3. Koordinationsinstrumente $\ldots \ldots \ldots \ldots \ldots \ldots \ldots \ldots \ldots \ldots \ldots, 111$

IV. Mikroökonomisch-empirische Konzepte und Hypothesen $\ldots \ldots \ldots \ldots .115$

\section{Kapitel 2}

Laterale Kooperation mit Forschungs- und Entwicklungsabteilungen -

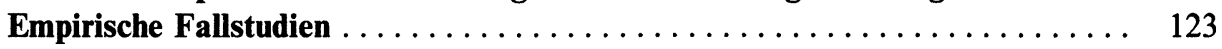

I. Querschnittsanalyse der empirischen Fallstudien ............... 124

1. Kooperationsebenen ........................... 124

2. Firmengemeinsame Merkmale und Strukturen lateraler Kooperationsbeziehungen ...................... 125

3. Firmengemeinsame Ursachen und Folgen von Kooperationskonflikten 127

4. Konfliktlösungsstrategien $\ldots \ldots \ldots \ldots \ldots \ldots \ldots \ldots \ldots \ldots \ldots \ldots$

II. Detailanalyse der empirischen Fallstudien ................. 131

1. Firmenspezifische Kooperationsprofile und -konflikte ......... 132

1.1. Fallstudie: Elektronik ....................... 132 
1.2. Fallstudie: Elektrotechnik $\ldots \ldots \ldots \ldots \ldots \ldots \ldots \ldots \ldots \ldots, 135$

1.3. Fallstudie: Pharma ......................... 136

2. Firmenspezifische Problem- und Konfliktfelder lateraler Kooperation von und mit FuE-Abteilungen . .................... 138

3. Evaluierung und Klassifizierung der Konfliktlösungsstrategien ..... 139

3.1. Fallstudie: Elektronik ............................. 140

3.2. Fallstudie: Elektrotechnik $\ldots \ldots \ldots \ldots \ldots \ldots \ldots \ldots \ldots, 140$

3.3. Fallstudie: Pharma $\ldots \ldots \ldots \ldots \ldots \ldots \ldots \ldots \ldots \ldots .142$

\section{Kapitel 3}

Mikroökonomisch fundiertes Kooperationsmanagement - Empirische Analyse und Gestaltung lateraler Kooperationsbeziehungen zwischen Forschung und Entwicklung, Marketing und Produktion

I. Kooperationsprofile und -konflikte - Ergebniszusammenfassung der

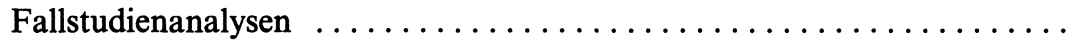

1. Fallstudiengemeinsame Merkmale und Strukturen der Organisation und Steuerung von Kooperationsprozessen $\ldots \ldots \ldots \ldots \ldots \ldots \ldots \ldots, 145$

2. Fallstudiengemeinsame Ursachen und Folgen von Kooperationskonflikten $\ldots \ldots \ldots \ldots \ldots \ldots \ldots \ldots \ldots \ldots \ldots, 148$

2.1. Kooperationsorganisation im Innovationsprozeß .......... 148

2.2. Konflikte im Kooperationsprozeß und Konfliktlösungsstrategien . 158

II. Organisation und Steuerung lateraler Kooperation mit FuE-Abteilungen Detailergebnisse der Fallstudienanalyse .................... 174

1. Fallstudie Elektronik ........................... 175

2. Fallstudie: Elektrotechnik ....................... 179

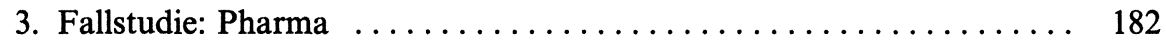

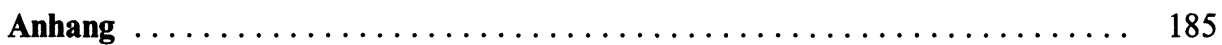

Enzyklopädisches Stichwort:

Mikroökonomische Organisationstheorie, Konzepte und Problemstellungen ...

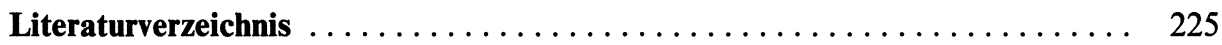

